\title{
SYMMETRY BREAKING IN EQUIVARIANT BIFURCATION PROBLEMS
}

\author{
M. J. FIELD AND R. W. RICHARDSON
}

\section{INTRODUCTION}

In both equivariant bifurcation theory [GSS, especially Chapter XIII] and physical theories of spontaneous symmetry breaking (for example, the Higgs-Landau theory [M]), there is the problem of determining the symmetries, stabilities and branching patterns for solutions of equations equivariant under a compact Lie group $G$. Very few general results and techniques are known for the analysis of this problem, though versions of a Maximum Isotropy Subgroup Conjecture have been conjectured, to the effect that generically all solution branches have maximal isotropy (see for example $[G, M])$. General results of this type are of particular interest for applications on account of the inherent complexity of the structure of isotropy subgroups, invariants and equivariants for $G$-representations. In this note, we announce several new results for the general study of the symmetries and branching patterns for a large class of $G$-equivariant bifurcation problems. In particular, we give new counterexamples to the Maximal Isotropy Subgroup Conjecture and present examples where one can get precise information on the branching patterns. Our methods also show that one can get quite detailed information on these problems without full knowledge of the $G$-equivariants. To simplify our exposition, we assume $G$ finite.

Let $V$ be a finite dimensional real Hilbert space and $G \rightarrow \mathbf{O}(V)$ be an absolutely irreducible representation of the finite group $G$. Let $G$ act on $V \times \mathbb{R}$ by $g \cdot(x, \lambda)=(g \cdot x, \lambda)$ and let $\mathscr{X}=$ $C_{G}^{\infty}(V \times \mathbb{R}, V)$ be the space of smooth $G$-equivariant maps of $V \times \mathbb{R}$ to $V$. Give $\mathscr{X}$ the $C^{\infty}$-topology; subsets of $\mathscr{X}$ are given the induced topology. Each $f \in \mathscr{X}$ defines a one-parameter family $\left(f_{\lambda}\right)_{\lambda \in \mathbb{R}}$ of equivariant vector fields on $V$. We have $f(0, \lambda)=$ $0, \lambda \in \mathbb{R}$. These are the trivial zeros of $f$. We study zeros of $f$ bifurcating off the trivial zeros. Now $D_{1} f(0, \lambda)=\sigma_{f}(\lambda) I d_{V}$,

Received by the editors January 8, 1989 and, in revised form, July 25, 1989. 1980 Mathematics Subject Classification (1985 Revision). Primary 58F14, 58F10. 
where $\sigma_{f}: \mathbb{R} \rightarrow \mathbb{R}$ is smooth. Normalizing, we may restrict attention to the subset $\mathscr{X}_{0}=\left\{f \in \mathscr{X} \mid \sigma_{f}(\lambda)=\lambda(\lambda \in \mathbb{R})\right\}$ of $\mathscr{X}$. If $f \in \mathscr{X}_{0},(0,0)$ is a bifurcation point of $f$. We study the germ of $f^{-1}(0)$ at $(0,0)$ for generic $f$. We give results that allow us to obtain detailed information on such germs for many pairs $(G, V)$.

The second named author would like to thank the Institute for Advanced Study, Princeton for partial support during the preparation of this paper.

\section{SOLUTION BRANCHES AND STABLE MAPS}

Let $f \in \mathscr{X}_{0}$ and let $\gamma=(\phi, \eta):[0, \delta] \rightarrow V \times \mathbb{R}$ be a $C^{1}$-arc. Then $\gamma$ is a solution branch for $f$ at $(0,0)$ if: (i) $f(\gamma(s))=0$ and $\gamma^{\prime}(s) \neq 0 \quad(s \in[0, \delta])$; and (ii) $\gamma(0)=(0,0)$. Two solution branches $\gamma, \rho$ are equivalent if (roughly) the germs of image $(\gamma)$ and image $(\rho)$ at $(0,0)$ are equal. Let $\Sigma(f)$ be the set of equivalence classes of solution branches for $f$ and let $[\gamma]$ be the equivalence class of $\gamma$. Clearly $G$ acts on $\sum(f)$. The solution branch $\gamma$ (or $[\gamma]$ ) is a branch of hyperbolic zeros if, for every $s \in(0, \delta]$, $\phi(s)$ is a hyperbolic zero of $f_{\eta(s)}$. We say that $\alpha \in \sum(f)$ is stable if there is a neighborhood $U$ of $f$ in $\mathscr{X}_{0}$ such that if $\left(\mathbf{f}_{t}\right)_{t \in[0,1]}$ is a continuous curve in $U$ with $\mathbf{f}_{0}=f$, then there is a continuous family $\left(\gamma_{t}\right)_{t \in[0,1]}$ of $C^{1}$-maps $[0, \delta] \rightarrow V \times \mathbb{R}$ such that each $\gamma_{t}$ is a branch of hyperbolic zeros of $\mathbf{f}_{t}$ and $\left[\gamma_{0}\right]=\alpha$. We say that $f \in \mathscr{X}_{0}$ is stable if $\Sigma(f)$ is a finite set of stable branches and if there is a neighborhood $U$ of $f$ such that $|\Sigma(f)|=|\Sigma(h)|$ for every $h \in U$. The set $\mathscr{S}=\mathscr{S}(G, V)$ of stable $f \in \mathscr{X}_{0}$ is a dense open subset of $\mathscr{X}_{0}[\mathrm{~F}$, Theorem 3.3].

\section{SYMMETRY BREAKING ISOTROPY TYPES}

If $x \in V$, the conjugacy class $\left(G_{x}\right)$ of $G_{x}$ in $G$ is the isotropy type of $x ; I(G, V)$ denotes the set of isotropy types for $(G, V)$ and $\tau=\left(G_{x}\right)$ is maximal if $G_{x} \neq G$ and if $G_{x} \varsubsetneqq G_{y}$ implies $G_{y}=G$. The isotropy type $\tau$ is symmetry breaking (resp. generically symmetry breaking) if there exists a nonempty open subset (resp. a dense open subset) $U$ of $\mathscr{S}$ such that, for every $f \in U$, there exists $\alpha \in \Sigma(f)$ such that $\left(G_{\alpha}\right)=\tau$.

Recall that a line $L=\mathbb{R} x$ in $V$ is an axis of symmetry of $G$ if $L=V^{G_{x}}$. If $L=\mathbb{R} x$ is an axis of symmetry, then $\left(G_{x}\right)$ is maximal and is generically symmetry breaking [GSS, volume 2, Chapter XIII, Theorem 3.3], [FR]. 
The following statement, now known to be false for certain $(G, V)$, is known as the Maximal Isotropy Subgroup Conjecture (MISC):

(MISC) An isotropy type $\tau \neq(G)$ is symmetry breaking if and only if it is maximal.

In all examples known to us, maximal isotropy types are generically symmetry breaking. But it is not known whether this is always so or even whether there need exist any symmetry breaking isotropy types. Roughly speaking, symmetry breaking maximal isotropy types have been considered as "expected" and symmetry breaking submaximal isotropy types have been considered as "exceptional." Our examples show, however, that symmetry breaking submaximal isotropy types are extremely common and should not be considered as unusual phenomena.

Example 1 [FR]. The MISC holds for the irreducible reflection groups $W\left(A_{k}\right), W\left(B_{k}\right), W\left(F_{4}\right)$ and all reflection groups in dimension $\leq 3$. For the reflection groups $W\left(D_{k}\right)(k \geq 4)$, there is exactly one submaximal isotropy type which is (generically) symmetry breaking.

\section{THE STABILITY THEOREM}

Let $P_{G}(V, V)$ (resp. $\left.P_{G}^{d}(V, V)\right)$ be the space of $G$-equivariant polynomial maps (resp. $G$-equivariant homogeneous polynomial maps of degree $d$ ) from $V$ to $V$ and let $P_{G}^{(d)}=\sum_{r \leq d} P_{G}^{r}(V, V)$. We say that $R \in P_{G}(V, V)$ is radial if $R(x)=p(\bar{x}) x(x \in V)$, where $p$ is an invariant polynomial; $R_{G}(V, V)=\sum_{d} R_{G}^{d}(V, V)$ is the space of radial equivariants. Let $d(G, V)$ be the smallest integer $d$ such that $R_{G}^{d}(V, V) \neq P_{G}^{d}(V, V)$. If $Q \in P_{G}(V, V)$, then $\mathscr{P}_{Q}$ denotes the vector field on the unit sphere $S$ of $V$ given by $\mathscr{P}_{Q}(x)=Q(x)-(Q(x) \mid x) x(x \in V)$. Note that $\mathscr{P}_{Q+R}=\mathscr{P}_{Q}$ for all $R \in R_{G}(V, V)$.

Let $Q \in P_{G}^{d}(V, V)$ and let $\mathscr{Z}\left(\mathscr{P}_{Q}\right)$ be the zero set of $\mathscr{P}_{Q}$; if $a \in \mathscr{Z}\left(\mathscr{P}_{Q}\right)$, then $Q(a)=\mu_{a} a$, with $\mu_{a} \in \mathbb{R}$. For every $\kappa \in \mathbb{R}$, we define $Q^{\kappa} \in \mathscr{X}_{0}$ by $Q^{\kappa}(x, \lambda)=\lambda x-Q(x)+\kappa\|x\|^{2} x$. For $a \in \mathscr{Z}\left(\mathscr{P}_{Q}\right), \kappa \in \mathbb{R}$, define $\phi_{a}^{\kappa}:[0,1] \rightarrow V \times \mathbb{R}$ by $\phi_{a}^{\kappa}(s)=$ $\left(s a, \mu_{a} s^{d-1}-\kappa s^{2}\right)$; then $\phi_{a}^{\kappa}$ is a solution branch of $Q^{\kappa}$.

Theorem 1. Let $Q \in P_{G}^{d,(G, V)}(V, V)$ and let a be a hyperbolic zero of $\mathscr{P}_{Q}$. Then for all but finitely many values of $\kappa,\left[\phi_{a}^{\kappa}\right]$ is 
stable. If all zeros of $\mathscr{P}^{Q}$ are hyperbolic, then $Q^{\kappa}$ is stable for all but finitely many values of $\kappa$.

Theorem 1 is proved by a straightforward blowing-up and perturbation argument.

\section{THE VANISHING AXIS THEOREM}

Let $H$ be a subgroup of $G$ such that $(H, V)$ is absolutely irreducible and such that $d(G, V)=d(H, V)$. A line $L=\mathbb{R} x$ in $V$ is a vanishing axis of symmetry (VAS) for $H$ if (i) $L$ is an axis of symmetry for $G$; (ii) the isotropy type $\left(H_{x}\right) \in I(H, V)$ is not maximal; and (iii) there exists $Q \in P_{G}^{d(G, V)}(V, V)$ such that $x /\|x\|$ is a hyperbolic zero of $\mathscr{P}_{Q}$. As a corollary of Theorem 1 , we have

Theorem 2. If $L=\mathbb{R} x$ is a vanishing axis of symmetry for $H$, then $\left(H_{x}\right) \in I(H, V)$ is a submaximal symmetry breaking isotropy type and the MISC fails for $(H, V)$.

Identify the symmetric group $S_{k}$ with the group of all $k$ by $k$ permutation matrices. Let $\mathbb{Z}_{k}$ be the subgroup of $S_{k}$ generated by the cyclic permutation $(1,2, \ldots, k)$. Let $\mathbb{Z}_{2}^{k}$ be the group of all $k$ by $k$ matrices of the form $\operatorname{diag}( \pm 1, \ldots, \pm 1)$ and let $\left(\mathbb{Z}_{2}^{k}\right)^{\prime}=\left\{g \in \mathbb{Z}_{2}^{k} \mid \operatorname{det}(g)=1\right\}$.

Example 2. (a) Let $k \geq 4$, let $G=W\left(B_{k}\right)$, let $H=W\left(D_{k}\right)$ and let $x=(1, \ldots, 1,0)$. Then $\mathbb{R} x$ is a VAS for $H$. (b) Let $k \geq 4$, let $G=W\left(B_{k}\right)$, let $H=\mathbb{Z}_{k} \cdot\left(Z_{2}^{k}\right)^{\prime}$ and let $x=(1,1, \ldots, 1,0)$. Then $\mathbb{R} x$ is a VAS for $H$ and $H_{x}=\{1\}$. Thus the trivial isotropy type $(\{1\})$ is symmetry breaking for $\left(H, \mathbb{R}^{k}\right)$. (c) Let $G=\left(W\left(B_{3}\right)\right)$, let $H=\mathbb{Z}_{3} \cdot \mathbb{Z}_{2}^{3}$ and let $x=(1,1,0)$. Then $\mathbb{R} x$ is a VAS for $H$. This is the only example in $\mathbb{R}^{3}$ for which the MISC fails. (d) We have examples of subgroups $H$ of $W\left(B_{8}\right)$ of order 128 and $f \in \mathscr{S}\left(H, \mathbb{R}^{8}\right)$ such that $\sum(f)$ contains a branch of sinks with trivial isotropy. (e) Let $k \geq 3$, let $G=W\left(B_{k}\right)$ and let $H=\Gamma \cdot \mathbb{Z}_{2}^{k}$, where $\Gamma$ is a transitive subgroup of $S_{k}$. Assume $\Gamma \neq S_{k}, A_{k}$ (the alternating group), or, if $k=6$, is not isomorphic to $S_{5}$. Then there is a VAS for $\left(H, \mathbb{R}^{k}\right)$. 


\section{DETERMINANCY AND BRANCHING PATTERNS}

For $f \in \mathscr{Z}_{0}$, let $J^{d}(f)=j_{1}^{d}(f)(0,0) \in P_{G}^{(d)}(V, V)$ denote the $d$-jet of $f_{0}$ at 0 . Let $d>1$. We say that $(G, V)$ is $d$-determined if there exists a dense open subset $\mathscr{R}(d)$ of $P_{G}^{(d)}(V, V)$ such that if $f \in \mathscr{Q}_{0}$, then $f$ is stable if and only if $J^{d}(f) \in \mathscr{R}(d)$. By [F, Theorem 3.13], there exists $d \geq d(G, V)$ such that $(G, V)$ is $d$ determined; let $\delta(G, V)$ denote the smallest such integer $d$. Let $\mathscr{H}=\mathscr{H}(G, V)$ denote the set of all $Q \in P_{G}^{d(G, V)}(V, V)$ such that all zeros of $\mathscr{P}_{Q}$ are hyperbolic. As a second corollary of Theorem 1 , we have

Theorem 3. Assume that $d(G, V) \geq 3$ and that $\mathscr{H}$ is a dense open subset of $P_{G}^{d(G, V)}$. Then $d(G, V)=\delta(G, V)$.

Remark. If $d(G, V)=2$ and if $\mathscr{H}$ is a dense open subset of $P_{G}^{2}(V, V)$, then $\delta(G, V)$ equals 2 or 3 . For example, if $k$ is odd (resp. even), then $W\left(A_{k}\right)$ is 3-determined (resp. 2 determined).

Let $f \in \mathscr{S}(G, V)$. We call (the isomorphism class of) the $G$ set $\sum(f)$ the branching pattern of $f$. By the definition of stability, the branching pattern is locally constant on $\mathscr{S}$. If the hypotheses of Theorem 3 hold, it is often possible to get a precise description of the possible branching patterns for $(G, V)$.

Example 3. (a) For all of the reflection groups mentioned in Example 1, there is exactly one branching pattern. (b) Let $H$ be as in Examples 2(e). In low dimensional cases of this type, we have obtained detailed information on possible branching patterns. Typically, several different branching patterns may occur and, in particular, there are symmetry breaking isotropy types which are not generically symmetry breaking. (c) Let $G=\mathbb{Z}_{4} \cdot \mathbb{Z}_{2}^{4}$. It can be shown that there is an open subset $U$ of $\mathscr{S}^{4}$ such that if $f \in U$, then all branching is supercritical (forward), there are no branches of sinks, and there exist branches of (attracting) limit cycles branching off of the trivial solution.

Remarks. (a) It is possible to attach more information to the branching pattern, such as the direction of branching and the index of the hyperbolic zeros along each branch. Typically, these other invariants can vary on the open subsets of $\mathscr{S}$ where the branching pattern is constant. (b) Our main results carry over to the case where $G$ is a compact Lie group and we work in terms of flow-invariant $G$-orbits (as opposed to $G$-orbits of zeros). 


\section{REFERENCES}

[F] M. Field, Equivariant bifurcation theory and symmetry breaking, Dynamics and Differential Equations (to appear).

[FR] M. Field and R. Richardson, Symmetry breaking and the maximal isotropy subgroup conjecture for reflection groups, Arch. Rational Math. Mech. 105 (1989), 61-94.

[G] M. Golubitsky, The Benard problem, symmetry and the lattice of isotropy subgroups, Bifurcation Theory, Mechanics and Physics (C.P. Bruter et. al. eds.), D. Reidel, Dordrecht-Boston-Lancaster, 1983, pp. 225-257.

[GGS] M. Golubitsky, D. Schaefer and I. Stewart, Singularities and groups in bifurcation theory, Vol. 2, Applied Mathematical Sciences 69, Springer-Verlag, New York-Berlin-Heidelberg, 1988.

[M] L. Michel, Minima of Higgs Landau polynomials, Regards sur la Physique contemporaine (1980), 157-203, Edition CNRS, Paris, 1980.

Department of Mathematics, University of Sydney, Sydney, NSW 2006, Australia

Institute for Advanced Study, Princeton, New Jersey 08540

Current address (R. W. Richardson): Mathematics Research Section, IAS, Australian National University, Canberra, ACT 2601, Australia 